\title{
Evidence-based clinical practice parameter guidelines for the treatment of patients with metastatic brain tumors: introduction
}

\author{
Steven N. Kalkanis • Mark E. Linskey
}

Received: 7 September 2009/Accepted: 8 November 2009/Published online: 3 December 2009

(C) The Author(s) 2009. This article is published with open access at Springerlink.com

\section{Why clinical guidelines are desirable and important}

Properly understood and employed, evidence-based medicine $(\mathrm{EBM})$ is a tool of considerable value for medicine and neuro-oncology [1]. It provides a secure, scientificallydefensible base for clinical practice and practice improvement. However, pursued on an individual case-by-case basis, in purest form, it can be inefficient and time consuming, particularly for health providers with extremely busy clinical practices. Clinical guidelines based on the best evidence available, developed and regularly updated by subject matter experts, focusing on common and important clinical scenarios and questions, have the potential to be very desirable, useful, and efficient EBM tools for optimizing patient care.

Clinical practice parameter guidelines are defined as 'systematically developed statements to assist practitioner and patient decisions about appropriate health care for specific individual circumstances' [2]. An advantage of utilizing guidelines in clinical decision-making over sole reliance on randomized controlled trial (RCT) results, is that they take professional experience into account in an aggregate and more systematic manner, rather than on an individual or ad hoc basis [3]. Not only are more "experts" involved in the consensus process (diluting out outliers in opinion), but, in an evidence-based guidelines development process, the opinions solicited are the experts' opinions

\section{S. N. Kalkanis $(\square)$}

Department of Neurosurgery, Henry Ford Health System,

2799 West Grand Blvd, K-11, Detroit, MI 48202, USA

e-mail: kalkanis@neuro.hfh.edu; skalkan1@hfhs.org

M. E. Linskey

Department of Neurosurgery, University of California-Irvine Medical Center, Orange, CA, USA about the collected evidence in the literature, rather than simply their own personal opinions regarding the subject.

Multidisciplinary, evidence-linked clinical practice parameter guidelines, based on the most rigorous evidencebased methodology, offer the potential of reducing unexplainable variation in clinical practice while elevating the quality of patient care to the highest levels supported by the best available, and most up-to-date, evidence. They also have the potential to clearly point out where critical evidence "gaps" exist in areas important to clinical care that can then subsequently be filled by directed research planning and investment [4]. The goal of this guideline initiative is to optimize the care and outcome of our patients with brain metastases, by providing the most methodologically valid, evidence-linked treatment recommendations in a user-friendly and comprehensive manner, for real-world clinical scenarios encountered by clinicians and patients every day.

The healthcare policy implications of clinical practice parameter guidelines are very real and deserve the careful attention of both individual practitioners and our national medical professional organizations. Legislation efforts currently active in Washington include language focusing on development and inclusion of "appropriateness criteria" as a means of restricting medical care and reducing medical costs. They also include language focusing on the development and funding of comparative effectiveness research analyzing clinical effectiveness, and not just cost effectiveness. Each of these efforts will likely lead to a search for the best available clinical practice guidelines in key public health impact areas for the purpose of improving value for every healthcare dollar spent, as well as reducing cost through practice restriction. It is in our patients' interest, as well as our own as patient advocates, to ensure the availability of the highest quality guidelines 
based on a rigorous methodology, where strength of recommendations cannot exceed the strength of available evidence.

\section{Why brain metastases are an important area for clinical guideline development}

Secondary brain tumors, or brain metastases, are 4-5 times more common in incidence than primary brain tumors [5]. Furthermore, by definition, metastatic brain tumors imply primary cancers that can span the full gamut of organbased subspecialty clinical practice in medicine. Thus, from a public health impact, evidence-linked clinical practice parameter guidelines regarding the care of patients with metastatic brain tumors are likely to positively impact more patients, as well as more medical practitioners, than any other potential topic within neuro-oncology.

How can clinicians and patients best navigate through the myriad of treatment decision pathways for brain metastases?

According to the 2008 American Cancer Society Registry, approximately 1.4 million Americans are diagnosed with cancer every year [6] and up to $40 \%$ of these patients-over a half million people annually-will go onto develop one or more brain metastases [5]. While lung and breast are the most common tumor types, many malignancies metastasize to the brain. Treatment decisions must be individualized based on a complex array of both patient-specific and tumorspecific characteristics, especially since the number of therapeutic options has grown considerably over the past two decades.

A paradigm shift has occurred in the evolution of how we treat patients with brain metastases. No longer relegated to the realm of palliation with an expectation of a rapid neurological decline and inevitable neurological demise, patients with brain metastases now have a myriad of aggressive treatment options available to them, resulting in a longer life expectancy and better quality of life. With the use of markedly improved local control measures, patients are now often just as likely to succumb from their systemic disease, than from their brain tumor(s).

Whole brain radiation therapy (WBRT) has been the mainstay of metastatic brain tumor therapy for decades through the mid-1990s [7]. However, more recent data has highlighted the potential benefit of more aggressive local control measures involving surgical resection and stereotactic radiosurgery (SRS) in addition to WBRT [8, 9]. Even more recently, SRS alone, surgical resection and SRS, chemotherapy, and several cutting-edge investigational adjuvant therapies have come under consideration. The typical brain metastasis patient now encounters not only a general medical oncologist and a radiation oncologist, but also a neurosurgeon, and a neuro-oncologist.

\section{Guidelines for creating guidelines}

The ultimate validity of any guideline is critically related to three key factors: (1) the composition of the guideline panel and its process, (2) the identification and synthesis of the evidence, and (3) the method of guideline construction applied [10, 11]. The panel composition is crucial, both for ultimate acceptance of the guidelines by practicing physicians and for its critical influence on the recommendation step of guideline construction. Successful introduction of a guideline requires that all key disciplines contribute to its development to ensure ownership and support [12]. While sponsored by the American Association of Neurological Surgeons (AANS), the Congress of Neurological Surgeons (CNS), and the AANS/CNS Joint Tumor Section, this guideline initiative was pro-actively designed to be as inclusive of other related disciplines as possible to maximize its quality, acceptance, and potential impact. In addition to prominent neurosurgeons involved in surgical neuro-oncology and stereotactic radiosurgery, the multidisciplinary writing panel includes nationally recognized experts from radiation oncology, medical oncology, and neuro-oncology.

In order to maximize the quality of the identification and synthesis of evidence as well as the speed and efficiency of guideline development, the three organizations sponsoring this initiative contracted with McMaster University to facilitate the process over an anticipated twelve month timetable. The McMaster Evidence-Based Practice Center (EPC) is one of 15 EPCs federally funded through grants from the Agency for Healthcare Research and Quality (AHRQ) to assist in promoting quality of healthcare, reducing its cost, improving patient safety, decreasing medical errors, and broadening access to essential services by supporting outcomes studies, and implementing their findings through the dissemination of clinical guidelines in the U.S. Extremely expert in EBM techniques, and with an experienced staff and asset infrastructure in place, they have been instrumental in helping the author group expedite their work at the highest possible quality level, without any diminution of thoroughness or scientific rigor.

The choice of a rigorous evidence-linked recommendation methodology over an informal or formal consensus methodology was purposefully chosen to maximize rigor of the result and prevent over-stepping the strength of available evidence. Consensus guidelines can produce very valid and useful conclusions, however, one of their main weaknesses is that they often lead to recommendations 
even in areas where there is insufficient strength of evidence to recommend one approach over another. Truly evidence-linked guidelines are important for pointing out recommendations based on pertinent evidence, but they have an even more important function in allowing and preserving provider autonomy and flexibility in areas where insufficient evidence or strength of evidence exists to recommend standardization. The fear of "cook book" medicine resulting from the application of guidelines to clinical practice is best mitigated by adherence to strict evidence-linked methodology [4].

\section{How this process is different}

Not all guidelines are equivalent in quality. According to Woolf, there are three main methods of guideline development-informal consensus, formal consensus, and evidence-linked development [13]. From the standpoint of evidence based medicine (EBM), only the latter has evidentiary status for EBM decision-making. Indeed, the U.S. Institute of Medicine hopes to eventually restrict the use of the term "guideline" to systematically developed advisory statements created according to validated methodology [2]. Some consider consensus guidelines as intellectually suspect by reflecting expert opinion, which when promulgated as a "guideline," can formalize unsound practice [14]. Without strict adherence to systematic and validated methodology, panelists may be pooling ignorance as much as distilling wisdom [15]. Some guidelines are of questionable quality and there have been calls for guidelines on how to devise guidelines [16].

The U.S. National Guidelines Clearinghouse (NGC) [ http://www.guideline.gov] currently includes guidelines that have been formed through expert consensus alongside those based in systematic evidence-based methodology. It also includes guidelines that have been created by special interest and advocacy groups, subspecialty organizations, insurance companies, private consulting firms, cross-representative panels designed to include representatives from all potential stakeholders, and Evidence-Based Practice Centers (EPCs). Many of these guidelines conflict with one another, and there is currently no means of resolving or adjudicating these conflicts other than individual providers or oversight organizations making their own decision(s) as to which should take a position of supremacy or authority.

By clearly outlining our search process as facilitated by the McMaster University EPC, publishing our evidence tables, identifying the evidence and their rated strength, providing the linkage between identified evidence and each published recommendation, as well as publishing the rationale for the resultant strength of recommendation, this guideline effort represents the most rigorous, and transparently verifiable, clinical practice parameter guidelines effort for metastatic brain tumors yet achieved. Furthermore, this effort is more up-to-date at time of publication than many previous guidelines efforts, in large part due to the assistance of the McMaster University EPC in facilitating a 13 month start-completion timeline. Given that most guideline projects take over three years to complete, and have an average evidence obsolescence shelf-life of approximately five to seven years, our expedited timeline will hopefully lead to maximal clinical impact for a longer duration than previous efforts.

Lastly, we strongly believe that any set of comprehensive brain metastasis guidelines should not merely serve as a reflection of the best currently-available evidence, but should also shine a light on critical unanswered questions to develop new pathways for future treatments. We also designed these guidelines to support the academic mission of our colleagues in institutions around the country who are seeking to discover the next frontiers in neuro-oncology. To these ends, every chapter, whenever available, lists important needed areas of study and future directions for various clinical scenarios, and also outlines a current list of open clinical trials comparing one brain metastasis treatment modality to another-including treatments in radiotherapy, stereotactic radiosurgery, surgery and chemotherapy as well as treatments utilizing novel, emerging agents and combination therapies. Clinicians are encouraged to use these listings to support and enroll their patients in these important ongoing studies so that when these guidelines are updated in a few years, much more powerful evidence may exist for adopting one treatment regimen over another.

\section{Ranking clinical treatment scenarios by levels of recommendation}

As described in more detail in the following methodology chapter, every clinical treatment scenario involving brain metastases was highlighted and ranked by a level of recommendation, with Level 1 being the highest, and Level 3 the lowest, with sometimes no recommendations being made depending on the quantity and quality of the evidence. Rigorous and lively debate ensued between all of the authors, but ultimately every author on the writing panel agreed to all of the ultimate recommendations after a careful review of the evidence itself and the strength of the evidence. The panel's strict adherence to the two-step systematic review process, in collaboration with our McMaster EPC partners, highlights a critically important and unique feature of this effort. As might be expected, given its rapidly emerging role in the treatment of brain metastases over the past decade, the SRS recommendations engendered the most spirited discussions amongst our 
multidisciplinary panel. Nonetheless, each recommendation was carefully constructed to stay fully within the confines of the power of the evidence in a process fully supported and endorsed by all members of the panel. With a clear recognition of both their limitations and promise, these clinical treatment scenarios and recommendations have been organized into the following chapters:

I. Radiation therapy in newly-diagnosed brain metastases

II. Surgical resection in newly-diagnosed brain metastases

III. Stereotactic radiosurgery in newly-diagnosed brain metastases

IV. Chemotherapy in newly-diagnosed brain metastases

V. Re-treatment modalities for recurrent and/or progressive brain metastases

VI. The role of prophylactic anticonvulsants in brain metastases

VII. The role of steroid therapy in brain metastases

VIII. Novel and investigational therapies for brain metastases

Acknowledgments We would like to acknowledge the contributions and visionary leadership of Dr. Beverly Walters, and we would also like to acknowledge the contributions of the McMaster Evidencebased Practice Center (EPC), Dr. Parminder Raina (Director). Dr. Lina Santaguida (Co-Associate Director, Senior Scientist) led the EPC staff, which was responsible for managing the systematic review process, searching for and retrieving, reviewing, data abstraction of all articles, preparation of the tables and the formatting and editing of the final manuscripts.

Disclaimer of liability The information in these guidelines reflects the current state of knowledge at the time of completion. The presentations are designed to provide an accurate review of the subject matter covered. These guidelines are disseminated with the understanding that the recommendations by the authors and consultants who have collaborated in their development are not meant to replace the individualized care and treatment advice from a patient's physician(s). If medical advice or assistance is required, the services of a competent physician should be sought. The proposals contained in these guidelines may not be suitable for use in all circumstances. The choice to implement any particular recommendation contained in these guidelines must be made by a managing physician in light of the situation in each particular patient and on the basis of existing resources.

Disclosures All panel members provided full disclosure of conflicts of interest, if any, prior to establishing the recommendations contained within these guidelines.

Open Access This article is distributed under the terms of the Creative Commons Attribution Noncommercial License which permits any noncommercial use, distribution, and reproduction in any medium, provided the original author(s) and source are credited.

\section{References}

1. Linskey ME (2006) Evidence-based medicine for neurosurgeons: introduction and methodology. Prog Neurol Surg 19:1-53

2. Institute of Medicine, Committee to Advise the Public Health Service on Clinical Practice Guidelines (1990) Clinical practice guidelines: directions for a new program. In: Field MJ, Lohr KN (eds) National Academy Press

3. Maisonneuve H, Ojasoo T (1999) From the life-cycles of clinical evidence to the learning curve of clinical experience. J Eval Clin Pract 5(4):417-421

4. Linskey ME (2008) The emergence of clinical practice parameter guidelines in neuro-oncology: promise of utility tempered with caution. J Neurooncol 89(3):359-362

5. Gavrilovic IT, Posner JB (2005) Brain metastases: epidemiology and pathophysiology. J Neurooncol 75(1):5-14

6. American Cancer Society (2008) Cancer facts \& figures 2008. http://www.cancer.org/docroot/stt/content/stt_1x_cancer_facts_ and_figures_2008.asp

7. Patchell RA, Tibbs PA, Regine WF, Dempsey RJ, Mohiuddin M, Kryscio RJ et al (1998) Postoperative radiotherapy in the treatment of single metastases to the brain: a randomized trial. JAMA 280(17):1485-1489

8. Kondziolka D, Patel A, Lunsford LD, Kassam A, Flickinger JC (1999) Stereotactic radiosurgery plus whole brain radiotherapy versus radiotherapy alone for patients with multiple brain metastases. Int J Radiat Oncol Biol Phys 45(2):427-434

9. Andrews DW, Scott CB, Sperduto PW, Flanders AE, Gaspar LE, Schell MC et al (2004) Whole brain radiation therapy with or without stereotactic radiosurgery boost for patients with one to three brain metastases: phase III results of the RTOG 9508 randomised trial. Lancet 363(9422):1665-1672

10. Grimshaw JM, Russell IT (1993) Effect of clinical guidelines on medical practice: a systematic review of rigorous evaluations. Lancet 342(8883): 1317-1322

11. Grimshaw J, Russell I (1993) Achieving health gain through clinical guidelines. I: Developing scientifically valid guidelines. Qual Health Care 2(4):243-248

12. Grimshaw J, Eccles M, Russell I (1995) Developing clinically valid practice guidelines. J Eval Clin Pract 1(1):37-48

13. Woolf SH (1992) Practice guidelines, a new reality in medicine. II. Methods of developing guidelines. Arch Intern Med 152(5):946-952

14. Delamothe T (1993) Wanted: guidelines that doctors will follow. BMJ 307(6898):218

15. Scott EA, Black N (1991) When does consensus exist in expert panels? J Public Health Med 13(1):35-39

16. Jackson R, Feder G (1998) Guidelines for clinical guidelines. BMJ 317(7156):427-428 